\title{
$\beta$-Mercaptoethanol-modified ELISA for diagnosis of visceral leishmaniasis
}

Correspondence

Abdallah el Harith

harith17@Yahoo.com

Received 25 March 2006

Accepted 18 May 2006

\author{
Elfadil M. Abass, ${ }^{1}$ Durria Mansour, ${ }^{1}$ Mohamed el Mutasim, ${ }^{1}$ Muna Hussein ${ }^{2}$ \\ and Abdallah el Harith $^{3} \dagger$ \\ ${ }^{1}$ Ahfad University for Women, PO Box 167, Omdurman, Sudan \\ ${ }^{2}$ Omdurman Hospital for Tropical Diseases, Omdurman, Sudan \\ ${ }^{3}$ Dutch Ministry of Foreign Affairs/Mission to Ahfad University for Women, Omdurman, Sudan
}

\section{INTRODUCTION}

Despite its high reliability for the diagnosis of visceral leishmaniasis $(\mathrm{VL})$, marginal titres are occasionally recorded with the direct agglutination test (DAT) in non-VL patients (Harith et al., 1988). Further improvements in test procedures, including the incorporation of $\beta$-mercaptoethanol $(\beta-\mathrm{ME})$ in antigen processing and urea for denaturing non-specific agglutinating antibodies, have improved test sensitivity and specificity (Harith et al., 1995). As an alternative to the conventional water-soluble antigen, an intact promastigote antigen has been evaluated in ELISA for the detection of anti-Leishmania IgG antibodies (Mohammed et al., 1985). Possibly due to the crude nature of the antigen employed, difficulties were encountered, impeding the reproducibility of VL diagnosis. In this study, we have combined the use of promastigote surface antigen(s) that are favourably modified (cleaved or unfolded) by $\beta$-ME, as demonstrated earlier for DAT, with defined serum immunoglobulins described for ELISA, to further optimize the diagnosis of VL in laboratories with limited facilities.

†Present address: Wijngaard 155, 8212 CJ Lelystad, the Netherlands. Abbreviations: DAT, direct agglutination test; $\beta$-ME, $\beta$-mercaptoethanol; $\mathrm{VL}$, visceral leishmaniasis.

\section{METHODS}

Strains and culture conditions. Leishmania donovani strain MHOM/68/1-S (an isolate from a VL case in Sudan), obtained from the Laboratory of Tropical Hygiene, Amsterdam, the Netherlands, was used throughout this study.

The strain was maintained by in vitro subculturing at 7-8 day intervals at $26^{\circ} \mathrm{C}$ in liver infusion tryptose (LIT)/haemin medium (Sadigursky \& Brodskyn, 1986). Promastigote mass production for antigen preparation was carried out by inoculating LIT/haemin-maintained parasites into RPMI 1640 medium supplemented with $5 \mathrm{mM}$ HEPES, $100 \mathrm{IU}$ penicillin $\mathrm{ml}^{-1}, 100 \mu \mathrm{g}$ streptomycin $\mathrm{ml}^{-1}$ and $10 \%$ heat-inactivated fetal calf serum. Incubation of the cultures was at $26^{\circ} \mathrm{C}$.

Patients and samples. Serum, plasma or whole-blood-spotted filter-paper specimens from subjects with the following conditions were included in the study:

Group A, proven VL $(n=40)$ from Gedaref, Eastern Sudan. VL was diagnosed by either bone-marrow or lymph-node aspiration (Kassab Treatment Centre, Gedaref State, Eastern Sudan).

Group B, high suspicion for VL $(n=145)$. Patients in this group manifested typical clinical signs and symptoms of VL, with negative diagnosis for malaria, typhoid and tuberculosis (Omdurman Hospital for Tropical Diseases, and Kassab Treatment Centre, Gedaref State, Eastern Sudan). In this patient group, the positive DAT results $(\geqslant 1: 3200)$ justified positive VL diagnosis. 
Group C, malaria $(n=28)$, Omdurman Hospital for Tropical Diseases.

Group D, typhoid ( $n=11)$, Omdurman Hospital for Tropical Diseases.

Group E, undetermined liver or spleen disorders $(n=22)$ with negative DAT $(\leqslant 1: 100)$, Omdurman Hospital for Tropical Diseases.

Group F, fever of unknown origin $(n=22)$. Patients in this group had a negative DAT titre $(\leqslant 1: 100)$, Omdurman Hospital for Tropical Diseases.

Group G, African trypanosomiasis ( $n=29)$, National Medical Research Institute, Khartoum, Sudan.

Group H, pulmonary tuberculosis $(n=28)$, Abuanja Academic Thorax Hospital, Omdurman, Sudan.

Group I, onchocerciasis $(n=10)$, Institute of Endemic Diseases, University of Khartoum, Sudan.

Group J, leukaemia $(n=8)$, Radiation and Isotopes Centre, Khartoum Central Hospital, Sudan.

Group K, apparently healthy non-endemic female students $(n=34)$, Omdurman Hospital for Tropical Diseases.

From 73 patients in groups $\mathrm{A}$ and $\mathrm{B}$, both serum- and blood-spotted filter-paper was collected. Only serum $(n=184)$ or plasma $(n=8)$ samples were collected from the patients in groups $\mathrm{C}-\mathrm{K}$. All samples were kept at $-20^{\circ} \mathrm{C}$.

Antigen processing and execution of serological tests. The antigen for DAT was prepared as described by Harith et al. (1995). The Coomassie Blue-stained promastigote suspension was washed and finally resuspended in $1.2 \%(\mathrm{v} / \mathrm{v})$ formaldehyde/citrate/saline as $10 \times$ concentrated $\left(5 \times 10^{8}\right.$ promastigotes $\left.\mathrm{ml}^{-1}\right)$ stock antigen.

The antigen for $\beta$-ME ELISA was processed as described above for DAT, with the exception of the Coomassie Blue staining. The intact promastigote antigen so processed was fixed in formaldehyde/citrate/ saline solution $(1 \cdot 2 \%, \mathrm{v} / \mathrm{v})$ at $1.5 \times 10^{8} \mathrm{ml}^{-1}$. As control, a promastigote antigen suspension was processed in a similar manner, but without $\beta$-ME treatment. The water-soluble (strain 1-S) antigen for conventional ELISA was kindly provided by the Laboratory of Biomedical Research, the Royal Tropical Institute, Amsterdam, the Netherlands.

For executing ELISA and DAT, spotted-blood filter-paper samples were eluted in physiological saline (1:50 serum dilution) overnight at $4{ }^{\circ} \mathrm{C}$ (Harith et al., 1988). ELISA was performed according to the procedures reported by Voller et al. (1980), using intact or water-soluble promastigote antigen. After addition of substrate (5-aminosalicylic acid), the reaction was measured at a wavelength of $450 \mathrm{~nm}$ on a Titrek Multiscanner. In one experiment, the test was also read visually $(1-1.5 \mathrm{~h}$ ) by locating the highest sample dilution that still showed colour by comparison with the blank control well. The cutoff was defined as the mean absorbance value obtained in 34 sera from healthy subjects $+3 \mathrm{SD}$.

In the conventional ELISA, the water-soluble antigen (protein content equivalent to that of a homogenate of $3 \times 10^{7}$ sonicated promastigotes $\mathrm{ml}^{-1}$ ) was used in combination with optimal serum and anti-human IgG conjugate dilutions of $1: 1600$ or $1: 100000$. A cutoff value of $0 \cdot 50$ (mean of test readings obtained in 25 healthy subjects $+3 \mathrm{SD}$ ) was established.

Dilutions of the $\beta$-ME-treated or -untreated stock antigen suspensions were made in carbonate/bicarbonate coating buffer ( $\mathrm{pH} 9 \cdot 6$ ), from $1 \times 10^{5}$ to $5 \times 10^{7}$ promastigotes $\mathrm{ml}^{-1}$. A promastigote concentration of $2 \cdot 5 \times 10^{7} \mathrm{ml}^{-1}$ for the $\beta$-ME-treated or -untreated intact antigen, and serum and conjugate dilutions of $1: 12800$ or $1: 100000$ were found to be optimal for executing the test. Absorbance values of $\geqslant 0 \cdot 27$ (mean of test readings obtained in 34 healthy subjects $+3 \mathrm{SD}$ ) were considered to be ELISA positives with the $\beta$-ME-treated or -untreated antigen.

The DAT was executed according to the improved protocol, taking $1: 3200$ as the cutoff (Harith et al., 1995). For confirmation or exclusion of VL, all samples showing marginal DAT titres $(1: 1600$ $1: 3200$ ) were retested after treatment with $0.03 \mathrm{M}$ urea.

Data analysis. The sensitivity for each of three tests ( $\beta$-ME ELISA, DAT and conventional ELISA) was defined as the proportion of true positives correctly identified [agreed positives/(agreed positives + false negatives) $\times 100 \%$ ]. Specificity was defined as the proportion of true negatives correctly identified [agreed negatives/(false positives + agreed negatives) $\times 100 \%$. The paired sample $t$ test was applied to compare results obtained in ELISA with the $\beta$-ME-treated or -untreated intact antigens, and Pearson's coefficient of correlation was employed to assess the performance of whole-blood sampling in comparison with serum.

\section{RESULTS}

Of 40 confirmed VL patients tested by $\beta$-ME ELISA, 39 $(97.5 \%)$ had positive absorbance values in the range $0 \cdot 28-2 \cdot 44$. No cross-reaction was observed in sera from healthy controls $(n=34)$ or from patients with clinical conditions other than VL $(n=158)$, revealing $100 \%$ specificity (Table 1 ). In contrast to results obtained with $\beta$-ME ELISA in non-VL sera $(n=158)$, two patients with leukaemia and four others with African trypanosomiasis scored positive marginal titres $(1: 1600-1: 3200)$ in DAT. By incorporating urea treatment into the DAT protocol, titres in all four trypanosomiasis patients were reduced to negative values $(<1: 3200)$, but remained marginally positive $(1: 3200)$ in the two patients with leukaemia (results not shown).

In $65(44 \cdot 8 \%)$ of 145 suspected VL patients tested, both $\beta$-ME ELISA and DAT scored positive readings (Table 1 ). Three patients $(2 \cdot 1 \%)$ were positive in DAT but negative in $\beta$-ME ELISA. Despite the discrepancy, concordance between the two tests was $95 \cdot 6 \%$ among seropositives and $96 \cdot 3 \%$ among seronegatives.

By comparison with the untreated intact antigen, the $\beta$-MEtreated antigen showed slightly lower absorbance values in the non-VL patients. At a 1:100 serum dilution, eight nonVL sera scored absorbance values above the cutoff $(0 \cdot 27)$ with the untreated antigen, of which seven were negative with the $\beta$-ME-treated antigen. Eleven non-VL sera crossreacted at a $1: 800$ dilution with the untreated antigen; negative ELISA readings were recorded in all 11 with the $\beta$-ME-treated antigen $(P<0 \cdot 042)$. Seven non-VL sera (from VL suspects, tuberculosis patients or healthy individuals) gave positive results with the untreated antigen at a $1: 3200$ dilution; only four of these tested positive with the $\beta$-MEtreated antigen. The difference between the two antigen preparations was even more pronounced $(P=0 \cdot 017)$ in the leukaemic sera. At a 1:12 800 serum dilution, however, no 
Table 1. Performance of $\beta$-ME ELISA and DAT in patients with confirmed or clinical suspicion of VL and in individuals with other conditions

\begin{tabular}{|lcccc|}
\hline \multirow{2}{*}{$\begin{array}{l}\text { Group } \\
\text { tested }\end{array}$} & \multicolumn{2}{c|}{$\boldsymbol{\beta}$-ME ELISA } & & DAT \\
\cline { 2 - 5 } & $\begin{array}{c}\text { Frequency of } \\
\text { positive results } \dagger\end{array}$ & $\begin{array}{c}\text { Absorbance value } \\
(\text { mean } \pm \text { SD })\end{array}$ & $\begin{array}{c}\text { Frequency of } \\
\text { positive results } \dagger\end{array}$ & $\begin{array}{c}\text { Reciprocal } \\
\text { of titre }\end{array}$ \\
\hline VL & $97 \cdot 5 \%(39 / 40)$ & $1 \cdot 163 \pm 0 \cdot 651$ & $100 \%(40 / 40)$ & $3200-\geqslant 102400$ \\
VLS & $44 \cdot 8 \%(65 / 145)$ & $0 \cdot 666 \pm 0 \cdot 757$ & $46 \cdot 9 \%(68 / 145)$ & $100-\geqslant 102400$ \\
DC & $0 \%(0 / 158)$ & $0 \cdot 131 \pm 0 \cdot 042$ & $0 \%(2 / 158)$ & $100-3200$ \\
HC & $0 \%(0 / 34)$ & $0 \cdot 181 \pm 0 \cdot 044$ & $0 / 34)$ & $\leqslant 100-200$ \\
\hline
\end{tabular}

${ }^{\star}$ Abbreviations: VL, visceral leishmaniasis; VLS, VL suspects; DC, diseased controls; HC, healthy controls.

$\dagger$ Percentage true positive (number positive/number tested).

cross-reaction was recorded in any of the 78 non-VL sera tested with the treated or untreated antigen. Also, irrespective of the antigen preparation applied, no significant difference $(P>0 \cdot 269)$ in sensitivity was observed in $14 \mathrm{VL}$ sera.

Based on the respective cutoffs, the absorbance values obtained in $18 \mathrm{VL}$ sera with the $\beta$-ME-treated antigen were generally higher $(0 \cdot 19-1 \cdot 86)$ than those with the watersoluble antigen $(0 \cdot 25-0 \cdot 78)$.

Four VL samples $(22 \cdot 2 \%)$ tested positive with the $\beta$-MEtreated antigen but negative with the water-soluble antigen. By comparison with the water-soluble antigen, the $\beta$-MEtreated antigen therefore showed a relatively higher sensitivity for VL detection $(88 \cdot 9$ versus $72 \cdot 2 \%)$. At the same respective cutoffs, both antigen preparations gave negative absorbance values ( $\beta$-ME treated, $0 \cdot 06-0 \cdot 24$; water-soluble, $0 \cdot 10-0 \cdot 46)$ with all 65 non-VL sera tested, implying $100 \%$ specificity.

In $24 \mathrm{VL}$ whole-blood samples collected on filter paper, results of $\beta$-ME ELISA correlated favourably $(r=0 \cdot 711)$ with those of the corresponding sera (results not shown). Absorbance values of $0 \cdot 25-1 \cdot 31$ were recorded for filterpaper sampling compared to $0 \cdot 28-2 \cdot 44$ with serum. Regardless of sampling procedure, comparable negative absorbance values of $0 \cdot 08-0 \cdot 23$ were also recorded in all 49 non-VL sera tested.

By comparison with the negative control reactions observed in blank wells, 17 of $20 \mathrm{VL}$ sera ( $85 \%)$ developed (at $>1: 12800$ dilution) visible colour within $1-2 \mathrm{~h}$ of substrate addition (results not shown). At this dilution, however, no or hardly discernible coloration developed in the wells containing non-VL sera $(n=25)$; all these samples also gave negative DAT readings $(<1: 100)$.

Throughout the 2-month storage period $\left(\right.$ at $4{ }^{\circ} \mathrm{C}$ ), the reactivity of the formaldehyde-fixed antigen remained stable. Employing the same batch of $\beta$-ME-treated antigen suspension, comparable ELISA absorbance values of $0 \cdot 27$ $2 \cdot 35$ were obtained at days $0,30,45$ and 60 in 25 of $26 \mathrm{VL}$ samples evaluated (results not shown). Similarly consistent antigen performance, but in the negative range $(<0 \cdot 27)$, was also observed in 32 non-VL sera tested on the same four occasions. Microscopic examination of specimens from the $\beta$-ME-treated antigen at day 60 of the storage period revealed normal promastigote morphology and a homogeneous antigen suspension.

\section{DISCUSSION}

At a cutoff of $0 \cdot 27$ based on optimal test conditions with respect to antigen concentration and serum and conjugate dilutions, the $\beta$-ME-modified ELISA showed a sensitivity of $97 \cdot 5 \%$. Comparable sensitivity levels $(97 \cdot 2-100 \%)$ have been reported earlier for ELISA with the conventional water-soluble or intact promastigote antigen (Shiddo, 1995; Hommel et al., 1978; Mohammed et al., 1985). However, in a similar Sudanese VL population tested with a recombinant Leishmania antigen in a strip test or conventional ELISA format, much lower sensitivity $(67 \%)$ has also been reported (Burns et al., 1993; Zijlstra et al., 2001). However, despite employing the same antigen to execute ELISA and DAT in this study, the sensitivity of the former technique was slightly lower ( $97 \cdot 5$ versus $100 \%)$. Whether the negative ELISA reading obtained in one VL patient reflected our choice of the more commonly applied IgG ELISA will be answered in future trials with IgM for monitoring early infection.

In contrast to results obtained with DAT, no cross-reaction was observed in the 65 non-VL sera (including leukaemia and trypanosomiasis) tested against the $\beta$-ME-treated antigen, implying $100 \%$ specificity. However, regardless of the Leishmania antigen employed in previous studies, crossreactions in ELISA have been reported with African trypanosomiasis and malaria sera (Mohammed et al., 1985; Voller et al., 1980). Even by comparison with the current recommended techniques, namely the freeze-dried DAT kit, rK39 ELISA, rK39 strip test and urine latex agglutination (Burns et al., 1993; Zijlstra et al., 2001), $\beta$-ME ELISA showed higher reliability for differential VL diagnosis in the Sudan. In our opinion, this excellent specificity can 
only be explained by the favourable cleaving or unfolding effect exerted by $\beta$-ME on complex promastigote surface antigens, whereby specific epitopes are optimally exposed for interaction with the corresponding IgG anti-Leishmania antibodies.

The high agreement $(r=0 \cdot 711 ; P=0 \cdot 000)$ between results obtained by filter-paper (blood) and serum sampling, together with the advantage of visual reading of test results, imply good potential for successful application in laboratories with limited facilities. At a 1:12800 serum dilution, cross-reaction with non-VL samples is significantly reduced, allowing for better discrimination of VL samples. Further optimization of the storage conditions, and of the temperature and duration of elution, is required for filter-paper whole-blood samples, to correct for the lower sensitivity $(91 \cdot 7 \%)$.

The formaldehyde-fixed antigen in $\beta$-ME ELISA proved stable during the 2-month storage period. This advantage was further enhanced by the absence of microscopic alterations in promastigote morphology and antigen homogeneity.

\section{ACKNOWLEDGEMENTS}

We are grateful to Dr Gasim Badri, president of Ahfad University for Women, Dr Farouk Abdulaziz, dean of the School of Medicine, Ahfad University for Women, and Dr Abubaker Uro, head of the Ahfad Centre for Science and Technology, Ahfad University for Women, for their invaluable support and encouragement. The efforts of Dr M. Mukhtar and Dr W. el Amin, Institute of Endemic Diseases, University of Khartoum, in cryopreservation and maintenance of the Leishmania strains are greatly appreciated. Our thanks also go to Dr Abdelgadir Elkadaro, Omdurman Hospital for Tropical Diseases, Dr B. Gasim el Seed, Abuanja Academic Thorax Hospital, Omdurman, and the medical staff at Kassab Treatment Centre for their cooperation throughout this study.
This investigation received financial support from the Netherlands Ministry of Foreign Affairs and the World Health Organization for the East Mediterranean Region (grant no. EMRO/TDR-6/10,T 5/762/6).

\section{REFERENCES}

Burns, J. M., Shreffer, W. G., Benso, D. R., Ghalib, H. W., Badaro, R. \& Reed, S. G. (1993). Molecular characterization of a kinesin-related antigen of Leishmania chagasi that detects specific antibody in African and American visceral leishmaniasis. Proc Natl Acad Sci U S A 90, 775-779.

Harith, A. E., Kolk, A. H. J., Leewenburg, J., Muigai, R., Jelsma, T. \& Kager, P. A. (1988). Improvement of a direct agglutination test for field studies of visceral leishmaniasis. J Clin Microbiol 26, 1321-1325.

Harith, A. E., Chowdhury, S., Al Massum, A., Samiao-Santos, S., Karim, E., El Safi, S. \& Haque, I. (1995). Evaluation of cleaving agents other than trypsin in direct agglutination test for further improving diagnosis of visceral leishmaniasis. J Clin Microbiol 33, 1984-1988.

Hommel, M., Peters, W., Ranque, J., Quilici, M. \& Lanotte, G. (1978). The micro-ELISA technique in the serodiagnosis of visceral leishmaniasis. Ann Trop Med Parasitol 72, 213-218.

Mohammed, E. A. R., Wright, P. A., Kager, P. A., Laarman, J. J. \& Pondman, K. W. (1985). ELISA using intact promastigotes for immunodiagnosis of kala-azar. Trans R Soc Trop Med Hyg 79, 344-350.

Sadigursky, M. \& Brodskyn, C. I. (1986). A new liquid medium without blood or serum for culture of haemoflagellates. Am J Trop Med Hyg 35, 942-944.

Shiddo, S. A. (1995). Visceral leishmaniasis in Somalia: immunodiagnostic and epidemiological aspects. $\mathrm{PhD}$ thesis, Swedish Institute for Infectious Disease Control.

Voller, A., Bidwell, D. \& Barlett, A. (1980). Enzyme-linked immunosorbent assay. In Manual of Clinical Immunology, pp. 359-371. Edited by N. Rose \& H. Friedman. Washington, DC: American Society for Microbiology.

Zijlstra, E. E., Nur, Y., Desjeux, P., Khalil, E. A. G., El-Hassan, A. M. \& Groen, J. (2001). Diagnosing visceral leishmaniasis with the recombinant K39 strip test: experience from Sudan. Trop Med Int Health 6, 108-113. 